\title{
Integrated hand-powered centrifugation and paper-based diagnosis with blood-in/answer-out capabilities
}

\author{
Bowei $\mathrm{Li}^{\mathrm{a}}{ }^{\mathrm{a}, 1}$, Ji Qi ${ }^{\mathrm{a}, 1}$, Longwen $\mathrm{Fu}^{\mathrm{a}}$, Jinglong Han ${ }^{\mathrm{b}}$, Jaebum Choo ${ }^{\mathrm{c}, * *}$, Andrew J. deMello ${ }^{\mathrm{d}, * * *}$, \\ Bingcheng Lin ${ }^{\mathrm{e}}$, Lingxin Chen ${ }^{\mathrm{a}, \mathrm{f}, *}$ \\ ${ }^{a}$ CAS Key Laboratory of Coastal Environmental Processes and Ecological Remediation, Shandong Key Laboratory of Coastal Environmental Processes, Yantai Institute of \\ Coastal Zone Research, Chinese Academy of Sciences, Yantai, 264003, China \\ ${ }^{\mathrm{b}}$ School of Environment and Materials Engineering and College of Chemistry and Chemical Engineering, Yantai University, Yantai, 264005, China \\ ${ }^{\mathrm{c}}$ Department of Chemistry, Chung-Ang University, Seoul, 06974, South Korea \\ ${ }^{\mathrm{d}}$ Institute for Chemical and Bioengineering, Department of Chemistry and Applied Biosciences, ETH Zürich, Vladimir Prelog Weg 1, 8093, Zurich, Switzerland \\ ${ }^{\mathrm{e}}$ Dalian Institute of Chemical Physics, Chinese Academy of Sciences, Dalian, 116023, China \\ ${ }^{\mathrm{f}}$ School of Pharmacy, Binzhou Medical University, Yantai, 264003, China
}

\section{A R T I C L E I N F O}

\section{Keywords:}

Microfluidic paper-based device

$\mu \mathrm{PAD}$

On site diagnosis

Cancer biomarker

\begin{abstract}
A B S T R A C T
To date, 55\% of inhabitants of the developing world still live in rural regions and they have a very urgent need to improve the level of disease diagnosis, especially in resource-limited settings. Herein, we present a microfluidic system that centrifuges whole blood and quantifies contained biomarkers in a rapid, feasible and integrated way. Such a device provides a blood-in/answer-out capability and is small enough to be carried by any individual in any environment. The successful integration a hand-powered centrifuge and immunoassay unit within a rotational paper-based device allows for diagnostic application by untrained users and in environments where access to electricity cannot be assumed. In addition, the low cost $(\sim \$ 0.5)$, light weight and small instrumental footprint make the device ideally suited for rapid on-site detection. To validate the applicability of the system in a clinical diagnostic testing, we successfully perform enzyme-linked immunosorbent assay (ELISA) analysis of carcinoembryonic antigen and alpha fetoprotein from human blood samples. We expect that this powerful platform technology will provide the opportunities for point-of-care diagnosis in resource-limited settings.
\end{abstract}

\section{Introduction}

Human blood usually carries a large amount of health information. Blood analysis is an important way to extract biological information and modern medical diagnosis. For the analysis of biomarkers in serum, the presence of blood cells will affect the analysis results, so the analysis of serum has become a necessary way for blood biomarkers and disease diagnosis (Mielczarek et al., 2016). Efficient analysis of blood serum is a key to a range of diagnostic processes in both the developed and developing world. At a basic level, blood serum analysis involves two component steps, namely treatment (i.e. the extraction of serum from whole blood) and the analysis of the serum sample. The most widely accepted route to extracting serum from whole blood is via centrifugation. Although efficient, commercial centrifuges are expensive, powered by electricity and bulky. This unsurprisingly, poses significant problems for the deployment of rapid and low-cost diagnostics within the developing world, and thus there is an urgent (and currently unmet) need for effective but affordable diagnostic technologies for use in poor-resource settings (Cybulski et al., 2014; deMello, 2006; Gong and Sinton, 2017; Martinez et al., 2010).

The immunoassay method is highly specific, has strong antiinterference ability, and the immune response process is relatively simple and easy to implement. It currently has irreplaceable application effects and commercial value in biomarker analysis and medical

\footnotetext{
* Corresponding author. CAS Key Laboratory of Coastal Environmental Processes and Ecological Remediation, Shandong Key Laboratory of Coastal Environmental Processes, Yantai Institute of Coastal Zone Research, Chinese Academy of Sciences, Yantai, 264003, China.

** Corresponding author.

$* * *$ Corresponding author.

E-mail addresses: jbchoo@cau.ac.kr (J. Choo), andrew.demello@chem.ethz.ch (A.J. deMello), 1xchen@yic.ac.cn (L. Chen).

1 These authors contributed equally: Bowei Li, Ji Qi.
} 
diagnosis at this stage (Giri et al., 2016). Take malaria diagnosis as an example, immunoassay for malaria diagnosis has been used in developing countries for more than ten years (Kim et al., 2017). Moreover, the effective method for early diagnosis of tumors marker is immunoassay (Wu et al., 2007). Certainly, a cheap, flexible, and easy-to-operate analysis platform can further support the development of immunoassay in in resource-limited settings or home medical care.

Lateral flow analysis (LFAs) provides a rapid detection platform for immunoassay. This simple analysis method has been extensively developed for the preliminary detection of some diseases and physical conditions (Posthuma-Trumpie et al., 2009). It has been rapidly commercialized. The most common judgment on female pregnancy has been in wide use since the 1980s. In addition, the preliminary judgment of malaria protozoa in blood has also been applied in developing countries for nearly 20 years. LFAs provide a fast way for convenient medical diagnosis (Ligler and Gooding, 2019; Salentijn et al., 2018). However, the widely used LFAs analysis method can only provide qualitative analysis results. This method is suitable for the fast diagnosis of diseases such as malaria, HIV (HIV virus), dengue fever, and other infectious diseases (Gasperino et al., 2018). However, LFAs cannot provide quantitative information in terms of commercial applications. As people have more urgent needs for home-based and on-site precise medical-care in the future, more suitable technique are needed to achieve this goal.

Based on range of inherent features, such as low unit costs, reduced reagent consumption, small footprints and minimal control architecture (Hu et al., 2014; Mahato et al., 2017; Martinez et al., 2010), microfluidic paper-based analytical devices ( $\mu$ PADs) (Cate et al., 2015; Martinez et al., 2008b; Reboud et al., 2019) have received enormous attention due to their potential use in fields such as point-of-care diagnostics (Bhamla et al., 2017; Li et al., 2017; Martinez et al., 2010; Tenda et al., 2018), biochemical testing (Bai et al., 2018; Tian et al., 2016; Xia et al., 2016; Xu et al., 2016), food safety (Alamo Busa et al., 2016; Zhang et al., 2015) and environmental analysis (Ding et al., 2017; Meredith et al., 2016; Qi et al., 2018). Importantly, such systems can be used in conjunction with a range of detecting techniques, including electrochemical (Ding et al., 2016; Ge et al., 2012; Qi et al., 2019; Zhang et al., 2018), colorimetric (Li et al., 2014; Liu and Crooks, 2011), and fluorescence methods (Noor et al., 2013; Qi et al., 2018). Paper-based microfluidic devices have been shown to be especially useful in point-of-care diagnostic applications (Cheng et al., 2010; deMello, 2006; Hu et al., 2014; Li et al., 2012). For example, Li et al. reported an origami-based device incorporating electrical impedance spectroscopy to quantify HIV p38 antigen in human serum at concentrations as low as $300 \mathrm{fg} / \mathrm{mL}$ (Li and Liu, 2016). In addition, Fridley and co-workers developed a paper-based device for malaria diagnosis, integrating enzyme-linked immunosorbent assays (ELISA), sample preparation, washing and signal enhancement steps (Fridley et al., 2014). Song et al. also reported the development of a "volumetric bar-chart chip" that links oxygen production by catalase with the displacement of ink along embedded microchannels. Such an approach was shown to be especially useful for the rapid and point-of-care quantification of protein biomarkers in clinical samples (Song et al., 2012). Other novel technology developments have included the use of (dissolvable) fluidic time delays for performing multi-step assays (Lutz et al., 2013), and paper-based chemiluminescence ELISA for the analysis of cancer biomarkers in human serum (Wang et al., 2012). That said, although these advances have been significant, almost all such methods are limited by the need to separate serum from blood prior to analysis. Accordingly, there is an urgent need to realize serum isolation and analysis from whole blood analysis within poor-resource settings.

Though some research group have already done some work using whole blood to measure important biochemical signal indicator on microfluidic paper chips, but they mainly use red blood cell agglutination effect to realize passive serum separation (Pollock et al., 2012; Vella et al., 2012; Yang et al., 2012). Recently, Bhamla and co-workers creatively proposed a method to separate serum from blood through centrifugation and not through passive filtration. They reported the development of a low-cost $(\sim 20 \phi)$, light weight and hand-powered centrifuge (the "paperfuge), which achieves rotational speeds of 125 , $000 \mathrm{rpm}$ without the need for electricity (Bhamla et al., 2017). But this paperfuge still had restricted use in performing blood analysis and obtaining the diagnostic results directly. Inspired by this elegant but powerful technology, we herein report the design, fabrication and testing of the integrated paper-based diagnostic system able to centrifuge whole-blood samples and analyze biomarkers via sandwich immunoassays. Fully Integrated hand-power Centrifuges and Analysis paper-based microfluidic devices (FICA $\mu$ PADs) effectively integrate both the centrifuge and immunoassay units and engender blood-in-answer-out operation. As concept-of-proof demonstrations, carcinoembryonic antigens (CEA) and alpha fetoproteins (AFP) from human serum are quantified using our FICA $\mu$ PADs. To the best of our knowledge, this study represents the novel demonstration of blood immune analysis, and defines an important breakthrough for diagnostics in resource-limited settings. Significantly, and without any process optimization, the manufacturing cost of a single FICA $\mu \mathrm{PAD}$ device is no more than $\$ 0.5$.

\section{Experimental}

\subsection{Materials}

All standard proteins (carcinoembryonic antigen (CEA), alpha fetoprotein (AFP), capture antibody CEA, and AFP, HRP-labeled signal antibody CEA and AFP) were purchased from Shanghai Linc-Bio Science Ltd. Whatman No.1 chromatography paper $(46 \mathrm{~cm} \times 57 \mathrm{~cm})$ was obtained from GE Healthcare Worldwide (Pudong Shanghai, China). Glutaraldehyde, chitosan, and bovine serum albumin (BSA) were purchased from Sigma-Aldrich. Ultrapure water $(>18.2 \mathrm{M} \Omega$ ) was obtained from a Millipore water purification system. ZJ-1 blood collection tubes were purchased from Taixing Guxi Glass Instrument Factory (Taizhou Jiangsu, China) and medical tape was purchased from Taizhou Jingwei Medical Equipment Ltd. (Taizhou Jiangsu, China). 3,3',5,5'-tetramethylbenzidine was purchased from J\&K Scientific company (Beijing, China). A phosphate buffer solution (PBS) containing 0.05\% Tween-20 $(\mathrm{pH}=7.4)$ acted as the washing buffer.

\subsection{Fabrication of paper-based device}

The paper-based device was fabricated via wax printing technology, and has been described in detail elsewhere (Li et al., 2014). Briefly, the fluidic pattern was designed using Adobe illustrator software, and then printed directly onto Whatman No.1 chromatography filter paper using a XEROX Phaser 8560DN wax printer. The printed paper was then heated to $150{ }^{\circ} \mathrm{C}$ and cooled to room temperature after a period of $30 \mathrm{~s}$.

\subsection{Integration of rotational paper-based centrifugal microfluidic device}

As shown in Fig. 1A, FICA $\mu$ PADs were divided into three layers; the detection disc (pink), the washing disc (deep blue), and the centrifugal separation disc (light blue). A hollow rivet was passed through all layers, with its end being expanded to unite the three layers in a single assembly. $330 \mathrm{~mm}$ nylon cord was then sequentially passed through the circular hole to form a closed loop between the device and the handle. This entire assembly procedure is presented in Movie S1.

Supplementary data related to this article can be found at https://do i.org/10.1016/j.bios.2020.112282.

\subsection{ELISA experiments on FICA $\mu$ PADs}

The diagnostic assay was divided into two parts, namely serum centrifugation and analysis. A $\sim 40 \mu \mathrm{L}$ blood sample was collected by 


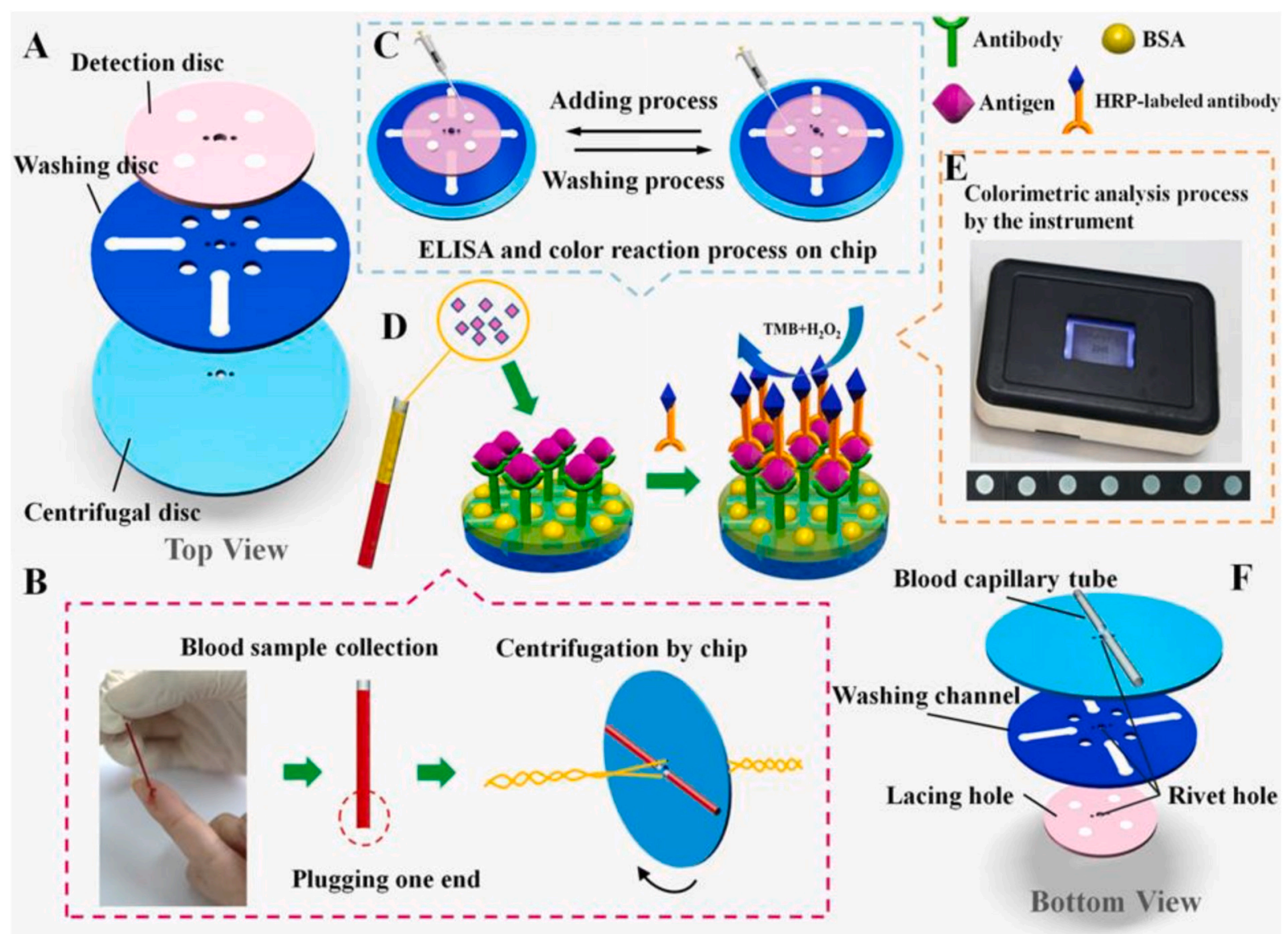

Fig. 1. Structure and operation of FICA $\mu$ PADs. (A) Exploded view of a fully integrated hand-powered centrifuges and analysis device (FICA $\mu$ PAD). (B) A finger prick blood sample is loaded and settled in a capillary tube and subsequently centrifuged. (C \& D) An ELISA assay is then performed, with an HRP-labeled secondary antibody catalyzing a 3,3',5,5'-tetramethylbenzidine (TMB) $-\mathrm{H}_{2} \mathrm{O}_{2}$ solution and generating a blue colored product (E) The generated signal is obtained using a portable reader. (F) A bottom view of a FICA $\mu \mathrm{PAD}$, highlighting the immuno-reaction region, the washing channel and blood collection tube. (For interpretation of the references to color in this figure legend, the reader is referred to the Web version of this article.)

puncturing a patient's fingertip with a $28 \mathrm{G}$ blood collection needle. One end of the capillary tube was sealed with glue while the other end was being left open. The capillary tube was then aligned with the center of the disc. As shown in the Movie S2, the device was then rotated to centrifuge the whole blood by simply pulling the handle. After $4.5 \mathrm{~min}$, serum was obtained in the upper layer of the tube. Subsequently, ELISA analysis of cancer markers CEA and AFP was then performed. First, valves on the reaction disk were closed and $5.0 \mu \mathrm{L}$ of $0.25 \mathrm{mg} / \mathrm{mL}$ chitosan added and allowed to naturally dry at room temperature for 15 min. Next, $5.0 \mu \mathrm{L}$ of $2.5 \%$ glutaraldehyde (PBS buffer, $\mathrm{pH}=7.4$ ) was added to each detection zone to promote aldehyde cross-linking on the paper surface. After developing the reaction for $2 \mathrm{~h}$, unreacted reagent was removed by six washes in PBS buffer. Next, $3.0 \mu \mathrm{L}$ of $1 \mu \mathrm{g} / \mathrm{mL}$ CEA and $3.0 \mu \mathrm{L}$ of $1 \mu \mathrm{g} / \mathrm{mL}$ AFP capture antibody were added to the two groups of immunozones in a diagonal direction. After $16 \mathrm{~min}$ at room temperature, excess antibodies not immobilized on the paper were removed by washing. To minimize non-specific adsorption on the paper surface, $5.0 \mu \mathrm{L}$ of $0.5 \%$ BSA solution was added to each immune zone to react $15 \mathrm{~min}$, and we would centrifuge the blood during this BSA blocking time. Subsequently, $3.0 \mu \mathrm{L}$ of user-defined concentrations of CEA and AFP standard solutions were added dropwise to the corresponding immunozones for $12 \mathrm{~min}$. Next, $3.0 \mu \mathrm{L}$ of $1 \mu \mathrm{g} / \mathrm{mL}$ HRP-labeled signal antibodies were added, incubated at room temperature during 20 $\mathrm{min}$ and then washed to remove the excess signal antibodies. Finally, 3 $\mu \mathrm{L}$ of $3,3^{\prime}, 5,5^{\prime}$-tetramethylbenzidine (TMB) $-\mathrm{H}_{2} \mathrm{O}_{2}$ solution was added to each test area to react $5 \mathrm{~min}$. The colorimetric results of CEA and AFP can be readout respectively by ELISA using our home-made portable device. In conclusion, the presented approach enabled the efficient centrifugation of whole-blood and identification of specific biomarkers through ELISA. The one sample is from our author (Ji Qi) and other human blood samples were randomly obtained from Yantai Affiliated Hospital of Binzhou Medical University. All the samples were tested by the hospital's method and make a comparison with our proposed method. All procedures and methods of this study were approved by the Committees for the Protection of Human Subjects at the Binzhou Medical University (Project identification code: IRB2019-60).

Supplementary data related to this article can be found at https://do i.org/10.1016/j.bios.2020.112282.

\subsection{Portable detection}

The portable colorimetric detector relies on light reflectance analysis, having dimensions of $11.8 \times 7.2 \times 2.0 \mathrm{~cm}$ ). The paper-based device is inserted into the drawer of the detector (Fig. S3), with color being directly converted to a digital output and result shown on screen (Li et al., 2014).

\section{Results and discussion}

\subsection{FICA $\mu P A D$ fabrication and assembly}

A schematic of a FICA $\mu$ PAD device is presented in Fig. 1 and Fig. S1. Briefly, the $\mu \mathrm{PAD}$ consists of three layers; termed the centrifugal separation layer (light blue disc, $95 \mathrm{~mm}$ in diameter), washing layer (deep 
blue disc, $83 \mathrm{~mm}$ in diameter) and reaction layer (pink disc, $55 \mathrm{~mm}$ in diameter). Layers are fixed using hollow rivets, with each layer free to independently rotate. As shown in Fig. S2, the centrifugal separation layer primarily acts as a carrier to hold capillary tubes during the centrifuging process and at the same time ensures no external crosscontamination during the ELISA analysis.

Blood samples are loaded into the capillary tubes and pasted on the back side of the centrifuge disc (Fig. 1F). The washing disc contains four fluidic paths (27 mm long), which are connected to four discrete detection sites on the top layer (diameter is $6 \mathrm{~mm}$ ). It should be noted that to ensure efficient centrifugation, two small holes symmetrically are located at a distance of $1 \mathrm{~mm}$ from the rivet. Nylon cord ( $66 \mathrm{~cm}$ long) is threaded through these holes and connected with a handle to facilitate the spinning of the paper chip (Fig. S2). The use of a handle ensures both comfortable for the operator and maintenance of stable rotational speed. The total weight of a FICA $\mu$ PADs including the paper chip, nylon cord and two hand-handles is $23.2 \mathrm{~g}$ (As displayed in Fig. S4). The entire assembly is shown in Movie S1. Two capillary tubes were used to collect whole blood samples. The blood capillary tube has an inner diameter of $1.1 \pm 0.1 \mathrm{~mm}$, an outer diameter of $1.5 \mathrm{~mm}$ and a length of $45 \mathrm{~mm}$. Each blood capillary tube carries about $40 \mu \mathrm{L}$ of whole blood; a volume enough for all subsequent analysis on the FICA $\mu$ PADs. After collection (Fig. 1B). The capillary tube was then positioned near the center of the disc and attached using medical tape. Under the action of sufficient centrifugal force, red blood cells were driven to the closed end of the tube, with serum remaining near the unsealed end. The entire assembly process is both facile and fast. After the ELISA assay was completed (Fig. 1C and D), the signal was measured using a portable paper-based colorimetric readout device (Fig. 1E). It is worth mentioning that the process relies on capillary forces and can be operated without any external power. The three-layer design integrates four rotating valve that provide control over the ELISA process. Moreover, it makes the integration of analysis and paper centrifuge units more coordinated and perfectly matching for this circular shaped chip. Therefore, it gives the device the ability to implement blood-in-answer-out analysis. In this study, as concept-of-proof demonstrations, two typical cancer biological biomarkers CEA and AFP were selected as model targets and analyzed on our $\mu$ PADs. The study also showed great potential in analyzing other target substances by changing their corresponding signal antibodies according to the real needs.

\subsection{Centrifugation}

The energy required to centrifuge a whole-blood sample originates from the manual application of force. As described previously, pulling and twisting the nylon cord converts translational energy into rotational energy (Bhamla et al., 2017) and provides sufficient centrifugal capacity to separate red blood cells from blood. The maximum speed of rotation of the centrifuge is given by previous report (Bhamla et al., 2017).

$\varphi_{\max }=\sqrt{\pi^{2}-4} \sqrt{\frac{F_{\mathrm{m}} L}{M_{\mathrm{d}} R_{\mathrm{d}}^{2}}}$

As illustrated in Fig. 2A, where $\varphi_{\max }$ is the maximum angular velocity, $\varphi_{\text {crit }}$ is the critical angular displacement, $L$ is the rope length and $R_{\mathrm{s}}$ is the radius of the rope. $F_{\mathrm{m}}$ represents the maximum value of the input force, $I$ is the moment of inertia, $\rho$ represents the average density of the entire rotating disc, $w_{\mathrm{d}}$ is the thickness of the rotating disc, $R_{\mathrm{d}}$ represents the radius of the rotating disc and $M_{\mathrm{d}}$ is the mass of the rotating disc. As illustrated in Fig. 2B, in the current system $F_{\mathrm{m}}$ is $9 \mathrm{~N}, L$ is 165 $\mathrm{mm}, m_{\mathrm{d}}$ is $3 \mathrm{~g}, R_{\mathrm{d}}$ is $47 \mathrm{~mm}$, and the maximum rotation speed achieves $10,939 \mathrm{rpm}$. The centrifugation process is shown in Fig. 2C and D and Movie S2. It is clearly observed that the blood was separated serum and red blood cells.

About rotational velocity calculation, we used the Simple Harmonic Oscillator (SHO) model theory of literature for calculation of rotational velocity of hand-powered paper-based centrifuge (paperfuge). Since for a paperfuge, we care about the maximum revolutions per minute (RPM), the literature reported formulas are simplified as:

$\varnothing_{\max }=\frac{\mathrm{L} \sqrt{\pi^{2}-2^{2}}}{2 R_{\mathrm{s}}} \sqrt{\frac{2 F_{\mathrm{m}} R_{\mathrm{s}}^{2}}{I L}}$
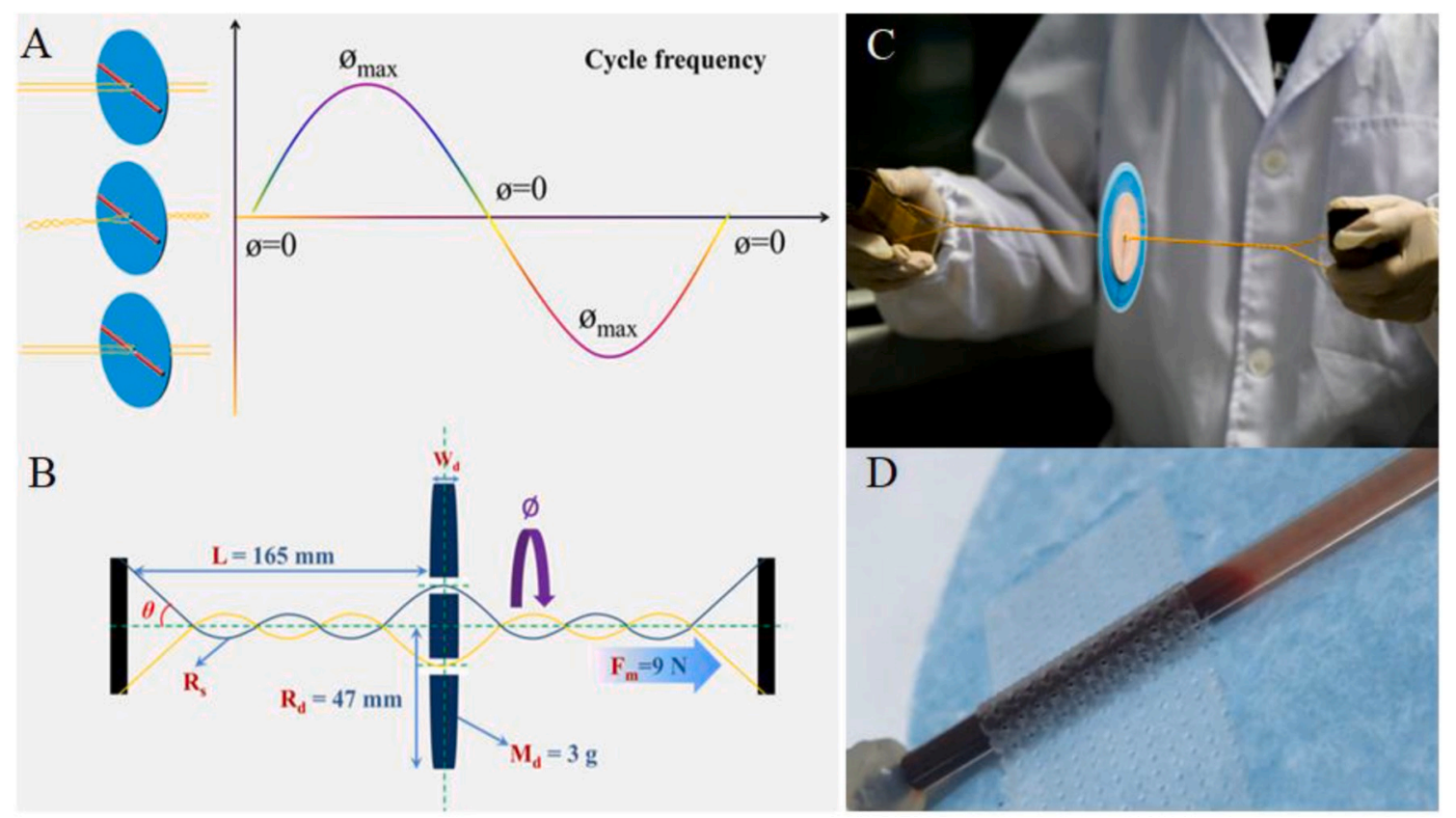

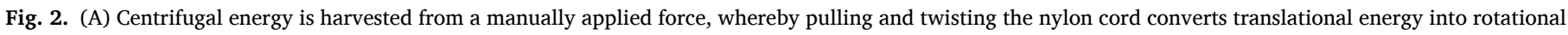

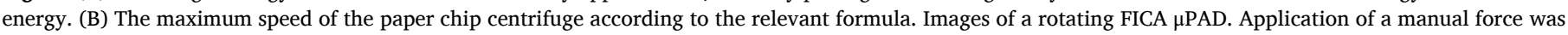

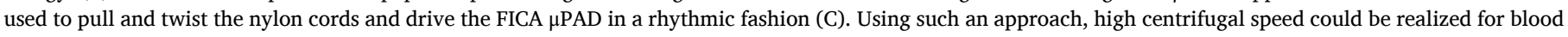
separation (D). 
$\mathrm{I}=\frac{\pi}{2} \rho \omega_{\mathrm{d}} R_{\mathrm{d}}^{4}$

Since the mass equation of the paperfuge was approximated by the mass equation of the cylinder, the formula eq. (3) could be simplified as:

$I=\frac{M_{\mathrm{d}} R_{\mathrm{d}}^{2}}{2}$

Thus, the RPM equation for the paperfuge can now be re-written as follows (the detail is in the supporting information):

$\varnothing_{\max }=\sqrt{\frac{\left(\pi^{2}-4\right) F_{\mathrm{m}} L}{M_{\mathrm{d}} R_{\mathrm{d}}^{2}}}$

In our prototyping FICA $\mu$ PADs, $\mathrm{F}_{\mathrm{m}}$ is $9 \mathrm{~N}, \mathrm{~L}$ is $165 \mathrm{~mm}, M_{\mathrm{d}}$ is $3 \mathrm{~g}, R_{\mathrm{d}}$ is $47 \mathrm{~mm}$.

$\varnothing_{\max }=\sqrt{\frac{\left(\pi^{2}-4\right) \times 9 \times 0.165}{0.003 \times 0.047^{2}}}=1145.566^{\circ} / s$

$R P M=\dot{\varnothing}_{\max } / 2 \pi=\left(\frac{1145.566^{\circ} / \mathrm{s}}{2 \pi}\right) \times 60 \mathrm{~s} / \min =10939 \mathrm{r} / \min$

\subsection{Detection process}

To evaluate whether the FICA $\mu \mathrm{PAD}$ is valuable in a clinical diagnosis, it must be capable of accepting human whole blood samples and generating qualitative results. Whole blood from capillary tubes was processed using the paper centrifuge, and serum collected from the upper layer was subsequently analyzed via enzyme-linked immunoassay on the same paper device.

As discussed, ELISA reactions typically involve multiple processes, including the incubation of multiple reagents, multi-step washes and non-specific site blocking using BSA. To address such demands, we designed and implemented rotational valves to allow for the orderly and effective manipulation of the immunoassay reagents. It is important to note that these valves are easy to control their "OFF" or "ON" states (Fig. 3 and Movie S3, S4) ensuring that the system is well suited for use in resource-limited setting.

Supplementary data related to this article can be found at https://do i.org/10.1016/j.bios.2020.112282.

To initiate the immune reaction, the washing disc was rotated by $45^{\circ}$ to close the channel (Fig. 3A) and prevent fluid from immune-zones flowing to the channels on the second layer. To make the whole process visual, we demonstrate this step with a green dye. Reagent was then added to the corresponding immuno-region on the detection disc. When washing was required, the washing disc was rotated $45^{\circ}$ to open the washing channel, with washing buffer rinsing away unreacted reagents along the washing channel by capillary action. Incubation of discrete volumes during the immunoassay and washes were realized by simple manipulation of the rotational valves. Next, a sandwich immunereaction was constructed on the FICA $\mu \mathrm{PAD}$ and colorimetric signals measured using our portable colorimetric readout device (Fig. S5) (Li et al., 2014). Furthermore, it should be noted that the FICA $\mu$ PAD can also be integrated with smart phone for assay detection (Martinez et al., 2008a; Shen et al., 2012; Thom et al., 2014). Mainly, the FICA $\mu$ PADs analysis platform realized the orderly manipulation of the immunoassay process through controlling the rotating valves.

\subsection{Optimization of the detection conditions}

The centrifugation time plays an important role in ensuring the collection of a sufficient volume of serum (Fig. 3D). Approximately 40 $\mu \mathrm{L}$ of whole blood in each capillary tube was stratified and separated at a high centrifugal speed and theoretical maximum could be achieved at $10939 \mathrm{rpm}$. Unsurprisingly, the quantity of serum obtained increased as a function of centrifugation time, with the amount of serum plateauing after $4 \mathrm{~min}$. Accordingly, and for the current experiments, we selected a centrifugation time of $4.5 \mathrm{~min}$. The average amount of serum collected
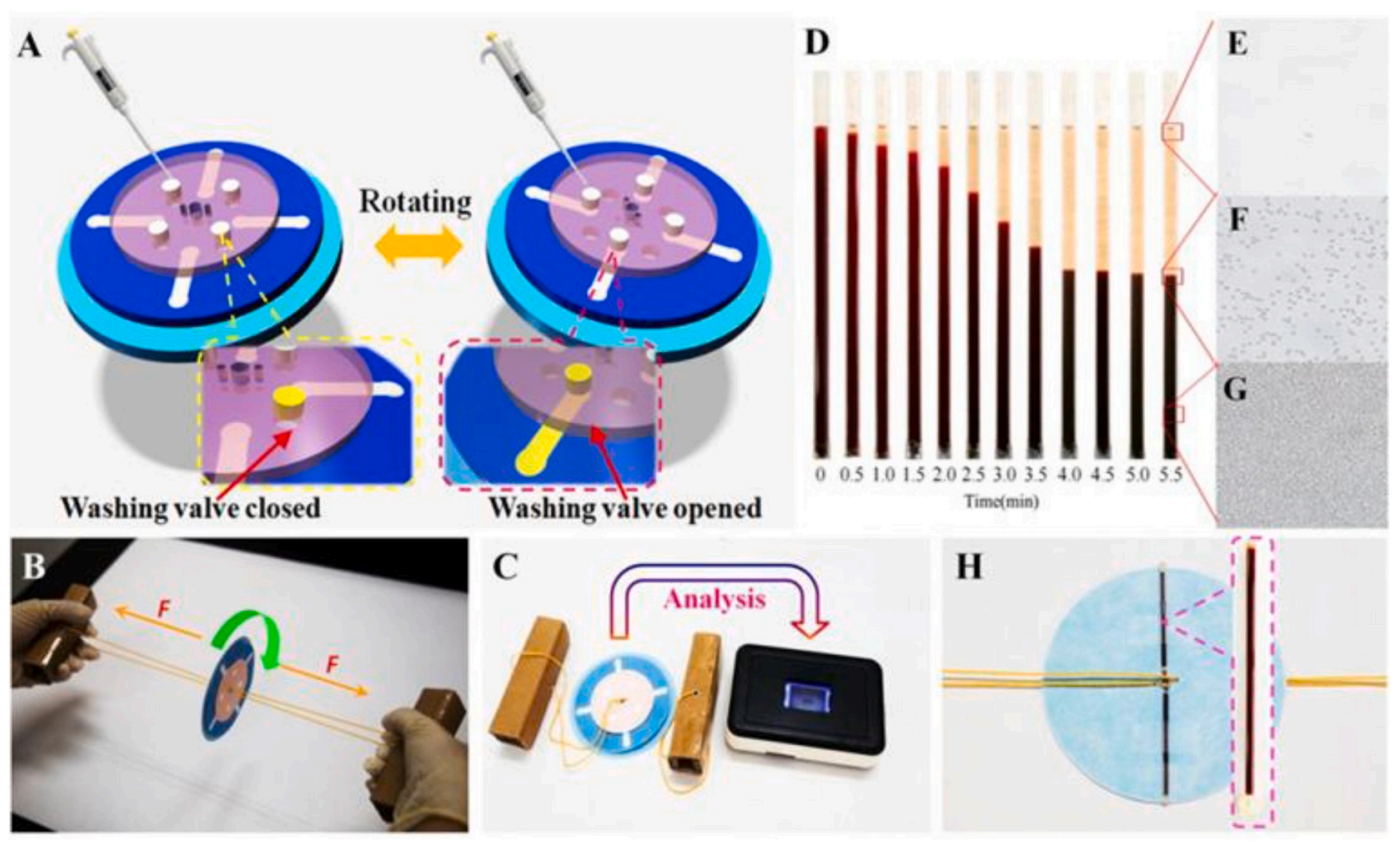

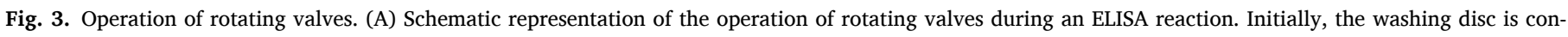

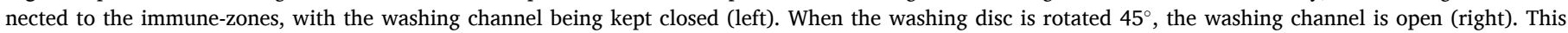

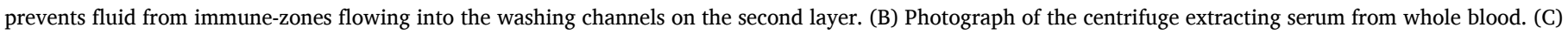

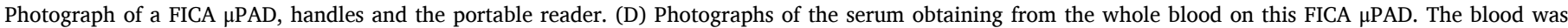

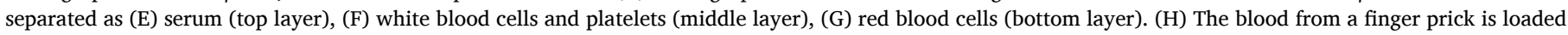

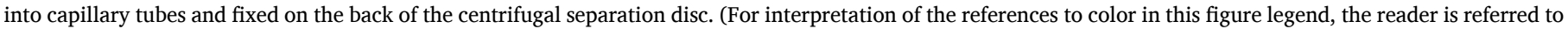
the Web version of this article.) 
from each centrifuge tube was about $20 \mu \mathrm{L}$; a quantity that easily satisfies the requirements for the assay (shown in Fig. S6).

ELISA performance with the FICA $\mu \mathrm{PAD}$ was found to be strongly dependent on the incubation time, analytical cost, $\mathrm{pH}$, and washing time efficiency. To this end, we optimized each parameter with a view to improve analytical performance. First, FITC-labeled CEA antibodies were used to study the optimal pipetting volume of solutions onto the FICA $\mu \mathrm{PAD}$, with different volumes $(0.5,1.0,1.5,2.0,2.5,3.0,3.5,4$ and $5.0 \mu \mathrm{L}$ ) of $50 \mathrm{ng} / \mathrm{mL}$ FITC-labeled CEA antibodies being added to the working zone. As presented in Fig. 4A, it was observed that the fluorescence originating from the paper zone was uniform when the pipetting volume was larger than $3 \mu \mathrm{L}$. Accordingly, a volume of $3 \mu \mathrm{L}$ was used in subsequent experiments. And every paper device's cost could be less than \$0.5 USD (Detailed information was shown in Table S1).

Next, the incubation time for the capture antibody and the HRPlabeled secondary antibody (10 $\mathrm{ng} / \mathrm{mL}$ CEA and AFP) was assessed. Fig. $4 \mathrm{~B}$ and $\mathrm{C}$, indicate that the intensity of the captured antibody for CEA and AFP stabilized after an incubation time of $16 \mathrm{~min}$ and the intensity of the HRP-labeled antibody for CEA and AFP stabilized after 20 min. Additionally, the incubation time for the CEA and AFP antigen was also investigated and presented in Fig. 4D, with signals reaching a plateau after an incubation time of $12 \mathrm{~min}$.

Assessment of the impact of $\mathrm{pH}$ is presented in Fig. 4E, with $\mathrm{pH}$ values varied between 6 and 9 . As can be seen, the signal reaches a maximum value at a $\mathrm{pH}$ of approximately 7.4, with further increases in $\mathrm{pH}$ leading to a dramatic decline in the intensity. Based on these measurements, a $0.01 \mathrm{~mol} / \mathrm{L}$ PBS buffer solution at a $\mathrm{pH}$ of 7.4 was used for all subsequent experiments.

It is well recognized that if non-specific binding proteins do not elute completely, the recorded ELISA signal (color) will be higher than expected, leading to the occurrence of false positives. To avoid this, we optimized the number of on-chip elutions, as illustrated in Fig. 4F and G, for FITC-labeled CEA antibodies. As can be observed, there was no appreciable variation in fluorescence or absorption after 6 elutions. Accordingly, 6 washes were used in all subsequent experiments.

\subsection{Analytical performance of FICA $\mu$ PADs}

We performed four sandwich HRP-labeled immunoassays using FICA $\mu$ PADs, to detect two kinds of tumor markers; CEA and AFP. The entire assay procedure is shown in Fig. 5C, with the primary immobilization steps following previously described reports (Ge et al., 2012; Liu et al., 2015). With the HRP-catalyzed TMB- $\mathrm{H}_{2} \mathrm{O}_{2}$ solution, the blue color was appeared and detected (Fig. S5). The paper detection sites was cut out from the disc and inserted into the portable reader for obtaining its color intensity. The detailed procedure is fully described in the Methods section (Movie S5).

Supplementary data related to this article can be found at https://do i.org/10.1016/j.bios.2020.112282.

The analytical performance was assessed under optimal conditions through application of two common tumor markers (CEA and AFP). Standard solutions of CEA $(1.0,3.0,5.0,7.0,10.0,15.0,20.0,30.0,40.0$, $100.0,300.0$, and $500.0 \mathrm{ng} / \mathrm{mL})$ and $\operatorname{AFP}(1.0,3.0,5.0,7.0,10.0,15.0$, 20.0, 25.0 35.0, 50.0, 100.0,300.0, and $500.0 \mathrm{ng} / \mathrm{mL}$ ) were directly introduced to immune-zones, with colorimetric signals being extracted using the paper-based colorimetric readout device. As shown in Fig. 5A and $\mathrm{B}$, the colorimetric signal (blue color) increased linearly with CEA and AFP concentration between $1.0 \mathrm{ng} / \mathrm{mL}$ to $40 \mathrm{ng} / \mathrm{mL}$ and $1.0 \mathrm{ng} / \mathrm{mL}$ to $35 \mathrm{ng} / \mathrm{mL}$, respectively. A simple linear regression analysis yielded fits of $y=23.089 x+864.437\left(R^{2}=0.982\right)$ for CEA and $y=29.588 x+$ $852.730\left(R^{2}=0.988\right)$ for AFP, respectively, where $y$ is the relative intensity and $x$ represent the marker concentration. When the CEA and AFP concentrations were more than 40.0 and $35.0 \mathrm{ng} / \mathrm{mL}$, respectively, the signal intensity reached the plateau and was not correlated with the
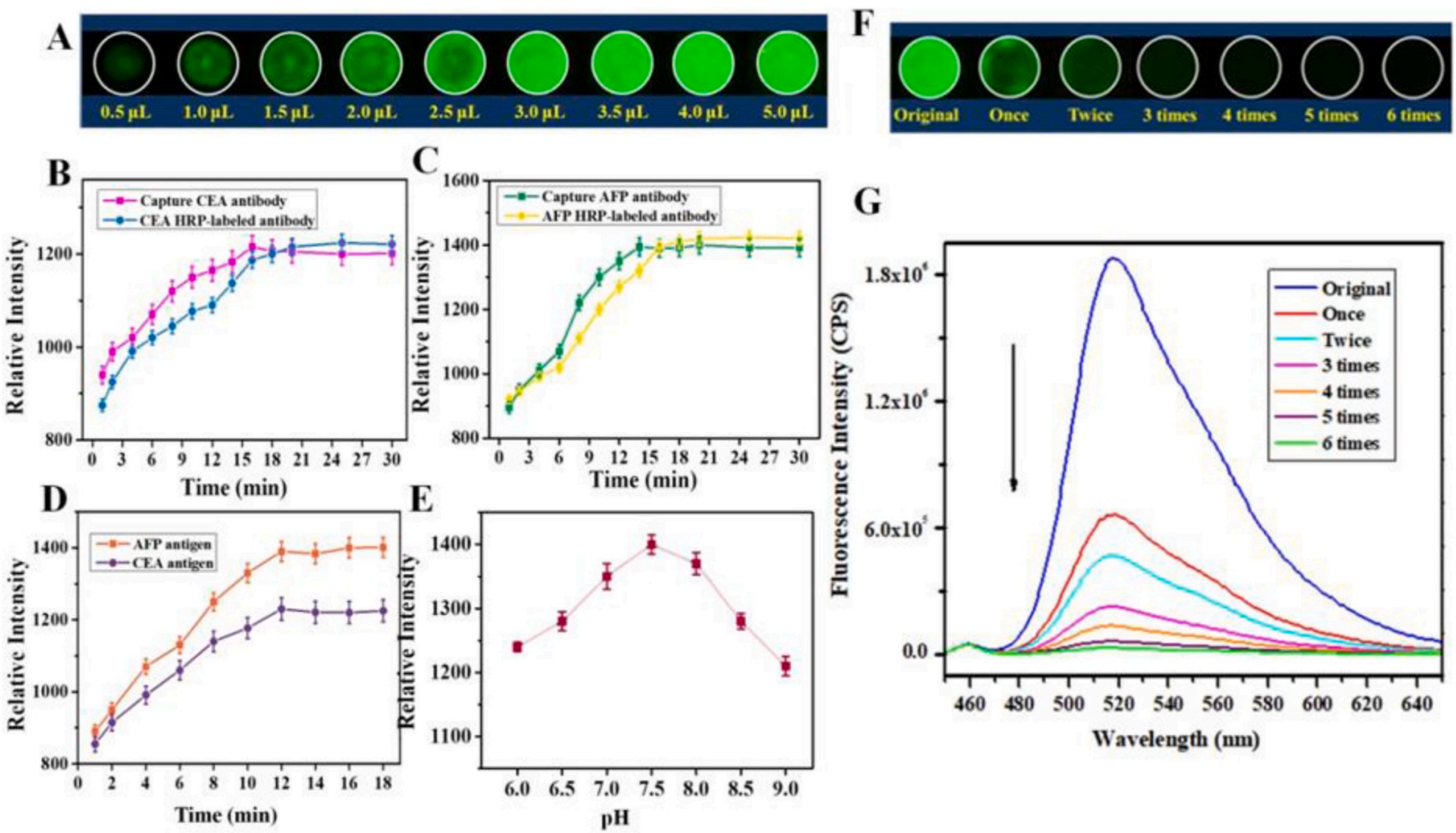

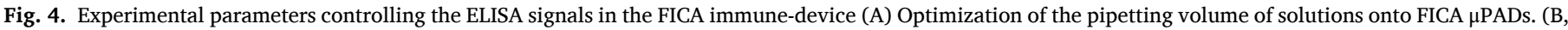

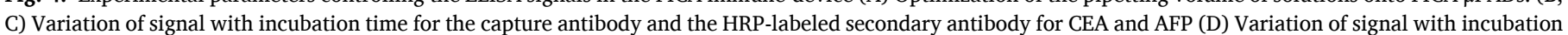

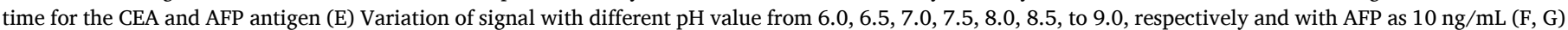
Images highlighting eluting effect of washes and the corresponding variations in the absorption spectrum of FITC-labeled CEA antibodies ( $\mathrm{n}=4$ ). 

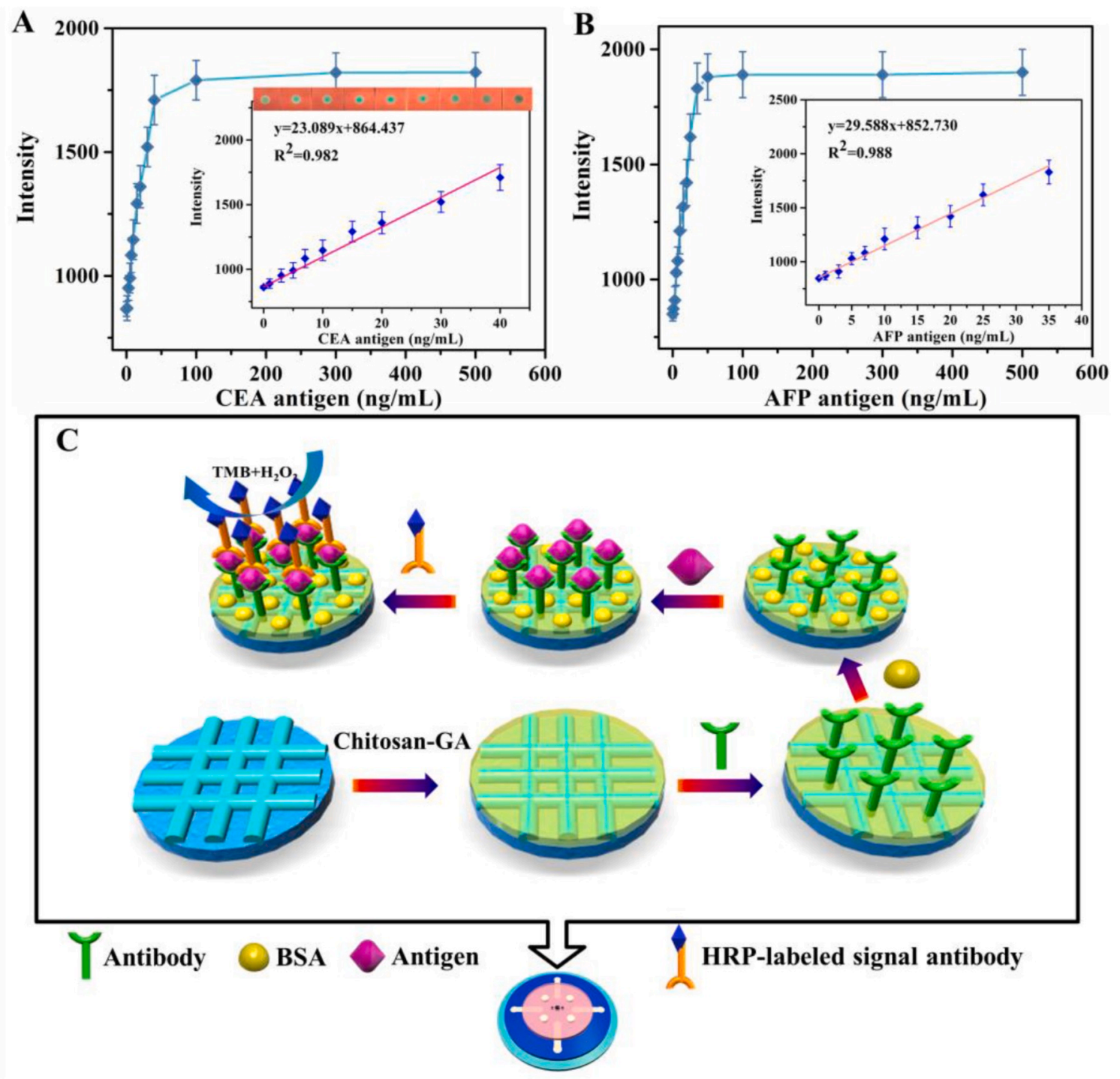

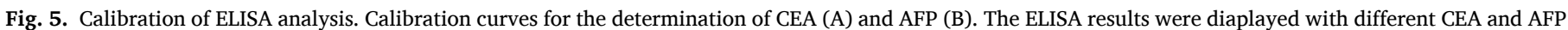

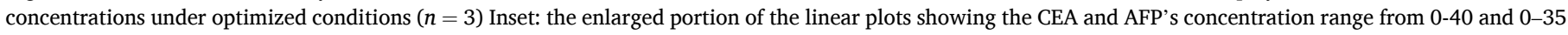
$\mathrm{ng} / \mathrm{mL}$, respectively. (C) Schematic diagram of the CEA and AFP immunoassay procedures using FICA $\mu$ PADs.

concentration of the target well. It is worthy notice that the quantitative determination curves could provide the diagnostic information. According to the LOD $=3 \sigma / S$, where $\sigma$ is the standard deviation of the intensity from blanks samples, $S$ is the slope of the linear fitting curve. Further analysis yielded concentration limits of detection of $360 \mathrm{pg} / \mathrm{mL}$ and $280 \mathrm{pg} / \mathrm{mL}$ for CEA and AFP, respectively. Critically, both values are significantly below the clinical threshold values (Erden et al., 2008; Laboria et al., 2010). As shown in Fig. S7, we can implement a real-time blood analysis in the outdoor and it displayed that the system has potential applications in resource-limited settings.

To further show that this method has good performance, include that the centrifugation can be expected when the device is used by different users, four non-skilled persons were selected to centrifuge the same blood sample using our FICA $\mu$ PADs. As shown in Fig. S8, all the serums were obtained successfully by these four persons. We also notice that the centrifuge finishing times are different by different individuals. This phenomenon can be explained. According to the formula Eq. (5), the obtained maximum angular velocity $\left(\varphi_{\max }\right)$ will be different. Therefore, the person with large pulling force may finish the centrifugation process faster than the person with small pulling force. However, all the persons will finish the blood centrifuge. As shown in Fig. 3D, the quantity of serum obtained will reach the plateau platform and keep constant. Therefore, the serum obtained is the same and does not depend on the skill of the user. Then we also tested the CEA and AFP concentrations in centrifuged serum samples by these 4 different persons. The results displayed that there is no significant difference in the measured results and RSD is lower than $5.2 \%$.

\subsection{Assessment of diagnostic application}

The performance of the described strategy was validated through determination of CEA in four human serums, with results being compared with data from traditional methods used in hospitals (Wilson, 2005). Based on the extracted standard curves, the quantitative results for CEA and AFP tumor markers are presented in Table 1. Analysis of both CEA and AFP samples report concentrations less than $5 \mathrm{ng} / \mathrm{mL}$, which is within the healthy range (Erden et al., 2008). Further, the data illustrate that the results provided by FICA $\mu$ PADs are consistent with those obtained using conventional hospital methods, with a relative standard deviation (RSD) between $4.03 \%$ and $4.98 \%$. Moreover, we also provided relevant experiment by samples spiked with larger concentrations of the two targeted analytes in Table S2. The data illustrated 
Table 1

Assay results from four human whole blood samples obtained with both FICA $\mu$ PADs and conventional hospital methods $(n=3$ ).

\begin{tabular}{|c|c|c|c|c|c|c|c|c|}
\hline \multirow[t]{2}{*}{ Analytes } & \multicolumn{4}{|c|}{ CEA (ng/mL) } & \multicolumn{4}{|l|}{$\mathrm{AFP}(\mathrm{ng} / \mathrm{mL})$} \\
\hline & Sample 1 & Sample 2 & Sample 3 & Sample 4 & Sample 1 & Sample 2 & Sample 3 & Sample 4 \\
\hline Proposed method & $1.12 \pm 0.1$ & $1.01 \pm 0.08$ & $2.01 \pm 0.11$ & $0.88 \pm 0.09$ & $1.31 \pm 0.09$ & $1.3 \pm 0.1$ & $2.98 \pm 0.08$ & $1.98 \pm 0.06$ \\
\hline Reference method & 1.32 & 0.81 & 2.15 & 0.94 & 1.11 & 1.15 & 3.10 & 1.82 \\
\hline RSD (\%) & 4.84 & 4.62 & 5.21 & 5.10 & 4.03 & 4.98 & 4.72 & 4.06 \\
\hline
\end{tabular}

that the FICA $\mu$ PADs display good performance in diagnostic applications.

\section{Conclusions}

In this work, we firstly presented a strategy to combine the function of centrifuging the blood sample and analyzing the specific biomarkers using sandwich immunoassay on the integrated paper-based microfluidic devices. The device has blood-in-answer-out capability and we can readout the results directly. The round three-layer design is perfectly suited for manual centrifugation, whilst integrated valves provide for effective and user-defined control of the ELISA reaction. To test the performance of our FICA $\mu$ PADs, CEA and AFP cancer biomarkers were selected as model targets, with experiments demonstrating concentration limits of detection of $360 \mathrm{pg} / \mathrm{mL}$ and $280 \mathrm{pg} / \mathrm{mL}$ for CEA and AFP, respectively. Based on these data, we believe that FICA $\mu$ PADs are highly promising and versatile tools for immunoassay diagnostics and due to their small size, simplicity and manual operation will bring significant utility and potential application in resource-limited settings.

\section{Declaration of competing interest}

The authors declare that they have no known competing financial interests or personal relationships that could have appeared to influence the work reported in this paper.

\section{CRediT authorship contribution statement}

Bowei Li: Conceptualization, Formal analysis, Investigation, Writing - original draft, Funding acquisition, Supervision. Ji Qi: Investigation, Formal analysis, Resources, Visualization, Writing - original draft. Longwen Fu: Investigation, Formal analysis, Resources. Jinglong Han: Investigation, Formal analysis. Jaebum Choo: Conceptualization, Visualization, Funding acquisition, Writing - review \& editing. Andrew J. deMello: Formal analysis, Visualization, Writing - original draft. Bingcheng Lin: Formal analysis, Writing - review \& editing. Lingxin Chen: Conceptualization, Writing - review \& editing, Funding acquisition, Supervision.

\section{Acknowledgments}

This work was financially supported by the National Key Research and Development Program of China (Grant No. 2016YFC1400702), the National Natural Science Foundation of China (Grant No. 41776110, 21876199, 21976209), Key Deployment Project of Centre for Ocean Mega-Research of Science, Chinese Academy of Sciences (COMS2019J01), and this work was partially supported by the government-wide R\&D Fund for the Research of Infectious Diseases in Korea (grant number HG18C0062) and the National Research Foundation of Korea (grant number 2019R1A2C3004375).

\section{Appendix A. Supplementary data}

Supplementary data to this article can be found online at https://doi. org/10.1016/j.bios.2020.112282.

\section{References}

Alamo Busa, L.S., Mohammadi, S., Maeki, M., Ishida, A., Tani, H., Tokeshi, M., 2016. Micromachines 7.

Bai, R., Li, L., Liu, M., Yan, S., Miao, C., Li, R., Luo, Y., Liu, T., Lin, B., Ji, Y., Lu, Y., 2018. Anal. Chem. 90, 5825-5832.

Bhamla, M.S., Benson, B., Chai, C., Katsikis, G., Johri, A., Prakash, M., 2017. Nat. Biomed. Eng. 1.

Cate, D.M., Adkins, J.A., Mettakoonpitak, J., Henry, C.S., 2015. Anal. Chem. 87, 19-41.

Cheng, C.-M., Martinez, A.W., Gong, J., Mace, C.R., Phillips, S.T., Carrilho, E., Mirica, K. A., Whitesides, G.M., 2010. Angew. Chem. Int. Ed. 49, 4771-4774.

Cybulski, J.S., Clements, J., Prakash, M., 2014. PLoS One vol. 9.

deMello, A.J., 2006. Nature 442, 394-402.

Ding, J., He, N., Lisak, G., Qin, W., Bobacka, J., 2017. Sens. Actuators, B 243, 346-352. Ding, J., Li, B., Chen, L., Qin, W., 2016. Angew. Chem. Int. Ed. 55, 13033-13037.

Erden, G., Barazi, A.O., Tezcan, G., Yildirimkaya, M.M., 2008. Scand. J. Clin. Lab. Invest. 68, 212-218.

Fridley, G.E., Le, H., Yager, P., 2014. Anal. Chem. 86, 6447-6453.

Gasperino, D.J., Leon, D., Lutz, B., Cate, D.M., Nichols, K.P., Bell, D., Weigl, B.H., 2018. Anal. Chem. 90, 6643-6650.

Ge, L., Yan, J., Song, X., Yan, M., Ge, S., Yu, J., 2012. Biomaterials 33, 1024-1031.

Giri, B., Pandey, B., Neupane, B., Ligler, F.S., 2016. Trac. Trends Anal. Chem. 79, 326-334.

Gong, M.M., Sinton, D., 2017. Chem. Rev. 117, 8447-8480.

Hu, J., Wang, S., Wang, L., Li, F., Pingguan-Murphy, B., Lu, T.J., Xu, F., 2014. Biosens. Bioelectron. 54, 585-597.

Kim, C., Hoffmann, G., Searson, P.C., 2017. ACS Sens. 2, 766-772.

Laboria, N., Fragoso, A., Kemmner, W., Latta, D., Nilsson, O., Luz Botero, M., Drese, K., O'Sullivan, C.K., 2010. Anal. Chem. 82, 1712-1719.

Li, B., Fu, L., Zhang, W., Feng, W., Chen, L., 2014. Electrophoresis 35, 1152-1159.

Li, B., Yu, L., Qi, J., Fu, L., Zhang, P., Chen, L., 2017. Anal. Chem. 89, 5708-5713.

Li, M., Tian, J., Al-Tamimi, M., Shen, W., 2012. Angew. Chem. Int. Ed. 51, 5497-5501.

Li, X., Liu, X., 2016. Adv. Healthc. Mater. 5, 1326-1335.

Ligler, F.S., Gooding, J.J., 2019. Anal. Chem. 91, 8732-8738.

Liu, H., Crooks, R.M., 2011. J. Am. Chem. Soc. 133, 17564-17566.

Liu, W., Guo, Y., Zhao, M., Li, H., Zhang, Z., 2015. Anal. Chem. 87, 7951-7957.

Lutz, B., Liang, T., Fu, E., Ramachandran, S., Kauffman, P., Yager, P., 2013. Lab Chip 13, 2840-2847.

Mahato, K., Srivastava, A., Chandra, P., 2017. Biosens. Bioelectron. 96, 246-259.

Martinez, A.W., Phillips, S.T., Carrilho, E., Thomas III, S.W., Sindi, H., Whitesides, G.M., 2008a. Anal. Chem. 80, 3699-3707.

Martinez, A.W., Phillips, S.T., Whitesides, G.M., 2008b. Proc. Natl. Acad. Sci. U. S. A. 105, 19606-19611.

Martinez, A.W., Phillips, S.T., Whitesides, G.M., Carrilho, E., 2010. Anal. Chem. 82, $3-10$.

Meredith, N.A., Quinn, C., Cate, D.M., Reilly III, T.H., Volckens, J., Henry, C.S., 2016. Analyst 141, 1874-1887.

Mielczarek, W.S., Obaje, E.A., Bachmann, T.T., Kersaudy-Kerhoas, M., 2016. Lab Chip $16,3441-3448$.

Noor, M.O., Shahmuradyan, A., Krull, U.J., 2013. Anal. Chem. 85, 1860-1867.

Posthuma-Trumpie, G.A., Korf, J., van Amerongen, A., 2009. Anal. Bioanal. Chem. 393, $569-582$.

Pollock, N.R., Rolland, J.P., Kumar, S., Beattie, P.D., Jain, S., Noubary, F., Wong, V.L., Pohlmann, R.A., Ryan, U.S., Whitesides, G.M., 2012. Sci. Transl. Med. 4, 152 ra129.

Qi, J., Li, B., Wang, X., Fu, L., Luo, L., Chen, L., 2018. Anal. Chem. 11827-11834.

Qi, J., Li, B., Zhou, N., Wang, X., Deng, D., Luo, L., Chen, L., 2019. Biosens. Bioelectron, $142,111533$.

Reboud, J., Xu, G., Garrett, A., Adriko, M., Yang, Z., Tukahebwa, E.M., Rowell, C., Cooper, J.M., 2019. Proc. Natl. Acad. Sci. U. S. A. 116, 4834-4842.

Salentijn, G.I.J., Grajewski, M., Verpoorte, E., 2018. Anal. Chem. 90, 13815-13825.

Shen, L., Hagen, J.A., Papautsky, I., 2012. Lab Chip 12, 4240-4243.

Song, Y., Zhang, Y., Bernard, P.E., Reuben, J.M., Ueno, N.T., Arlinghaus, R.B., Zu, Y., Qin, L., 2012. Nat. Commun. 3.

Tenda, K., van Gerven, B., Arts, R., Hiruta, Y., Merkx, M., Citterio, D., 2018. Angew. Chem. Int. Ed. 57, 15369-15373.

Thom, N.K., Lewis, G.G., Yeung, K., Phillips, S.T., 2014. RSC Adv. 4, 1334-1340.

Tian, T., Wei, X., Jia, S., Zhang, R., Li, J., Zhu, Z., Zhang, H., Ma, Y., Lin, Z., Yang, C.J., 2016. Biosens. Bioelectron. 77, 537-542.

Vella, S.J., Beattie, P., Cademartiri, R., Laromaine, A., Martinez, A.W., Phillips, S.T., Mirica, K.A., Whitesides, G.M., 2012. Anal. Chem. 84, 2883-2891.

Wang, S., Ge, L., Song, X., Yu, J., Ge, S., Huang, J., Zeng, F., 2012. Biosens. Bioelectron. 31, 212-218.

Wilson, M.S., 2005. Anal. Chem. 77, 1496-1502.

Wu, J., Fu, Z., Yan, F., Ju, H., 2007. Trac. Trends Anal. Chem. 26, 679-688. 
Xia, Y., Si, J., Li, Z., 2016. Biosens. Bioelectron. 77, 774-789.

Xu, B., Du, Y., Lin, J., Qi, M., Shu, B., Wen, X., Liang, G., Chen, B., Liu, D., 2016. Anal. Chem. 88, 11593-11600.

Yang, X., Forouzan, O., Brown, T.P., Shevkoplyas, S.S., 2012. Lab Chip 12, 274-280.
Zhang, Y., Zhang, L., Cui, K., Ge, S., Cheng, X., Yan, M., Yu, J., Liu, H., 2018. Adv. Mater. 30.

Zhang, Y., Zuo, P., Ye, B.-C., 2015. Biosens. Bioelectron. 68, 14-19. 